\title{
Esophagogastroduodenoscopy-induced angina bullosa hemorrhagica of the pharynx
}

\author{
Kenta Hamadaa, Yuka Obayashia, Yoshiro Kawaharab, Hiroyuki Okada ${ }^{a}$ \\ Okayama University Graduate School of Medicine, Dentistry and Pharmaceutical Sciences, Okayama, Japan
}

A 60-year-old man with a history of hypertension and hyperlipidemia underwent an esophagogastroduodenoscopy (EGD) for further inspection of a small brownish area in the postcricoid area detected during a laryngoscopy. The Valsalva maneuver was attempted using a transoral endoscope with a magnifying function; however, it was difficult to observe the postcricoid area because of a strong gag reflex. After the pharyngeal examination, angina bullosa hemorrhagica $(\mathrm{ABH})$ was observed in the uvula (Fig. 1) and the posterior wall of the hypopharynx (Fig. 2). The patient was followed up conservatively, because the lesions were mild and he was asymptomatic.

$\mathrm{ABH}$ is a benign lesion with subepithelial blisters filled with blood and not caused by a systemic or hemostatic disorder $[1,2]$. Mild trauma is the usual cause of a broken epitheliumconnective tissue junction, which results in superficial capillary bleeding $[1,2]$. The long-term use of inhaled steroids, hypertension, diabetes mellitus, and older age are considered as risk factors [2]. The differential diagnoses may include pemphigus, dermatitis herpetiformis, and bullous lichen planus [2]. Although $\mathrm{ABH}$ usually resolves spontaneously, tracheal intubation is required in extreme situations to protect the airway [3]. Therefore, gastroenterologists should be aware of EGD-induced $\mathrm{ABH}$.

Department of a Gastroenterology and Hepatology (Kenta Hamada,

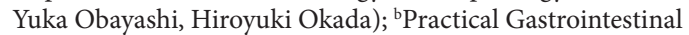
Endoscopy, Okayama University Graduate School of Medicine, Dentistry and Pharmaceutical Sciences, Okayama (Yoshiro Kawahara), Japan

Conflict of Interest: None

Correspondence to: Kenta Hamada, MD, Department of Gastroenterology and Hepatology, Okayama University Graduate School of Medicine, Dentistry and Pharmaceutical Sciences, 2-5-1 Shikata-cho, Kita-ku, Okayama 700-8558, Japan, e-mail: paosishou@yahoo.co.jp

Received 30 January 2020; accepted 3 February 2020; published online 27 March 2020

DOI: https://doi.org/10.20524/aog.2020.0473

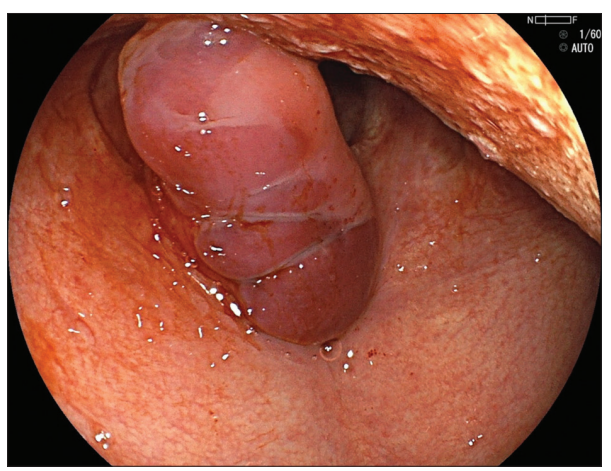

Figure 1 Endoscopic image of esophagogastroduodenoscopy-induced angina bullosa hemorrhagica in the uvula

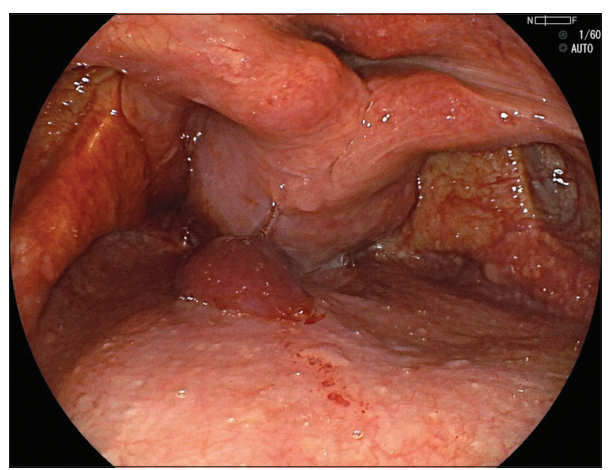

Figure 2 Endoscopic image of esophagogastroduodenoscopy-induced angina bullosa hemorrhagica in the posterior wall of the hypopharynx

\section{References}

1. Sato Y, Yokoyama K, Watanabe J, Nakamura A. Pharyngeal angina bullosa hemorrhagica due to EGD. Gastrointest Endosc 2018;87:1583-1584.

2. Hamada K, Yoshida A, Okada H. Esophagogastroduodenoscopyinduced angina bullosa hemorrhagica of the aryepiglottic folds and arytenoid. Clin Gastroenterol Hepatol 2019;17:A32.

3. Pahl C, Yarrow S, Steventon N, Saeed NR, Dyar O. Angina bullosa haemorrhagica presenting as acute upper airway obstruction. $\mathrm{Br} \mathrm{J}$ Anaesth 2004;92:283-286. 